\title{
Reply to the Letter to the Editor: Impact of Enhanced Recovery Program after Surgery in Patients Undergoing Pancreatectomy on Postoperative Outcomes: A Controlled before and after Study
}

\author{
Julie Perinel ${ }^{a, b} \quad$ Mustapha Adham ${ }^{a, b}$ \\ aService de Chirurgie Digestive, Hôpital Edouard Herriot, Hospices Civils de Lyon, Lyon, France; braculté de \\ Médecine de Lyon, Université Claude Bernard Lyon 1 (UCBL1), Lyon, France
}

Dear Editor,

We would like to thank Dr. Malgieri for his comments on our recently published study [1]. Our aim was to evaluate the impact of ERAS program on postoperative outcomes after pancreatectomy through a prospective controlled study. We compared two groups: the intervention hospital that implemented ERAS program and the control hospital that performed traditional care, and two periods: the pre-implementation and the post-implementation period.

Dr. Malgieri mentioned the lack of information in our study concerning the management of perioperative pain. Indeed, it is important to know the details of the intraand postoperative analgesic regimen in each group considering the impact of a multimodal opioid sparing strategy on postoperative outcomes [2].

In the non-ERAS cohort, as mentioned in our method, epidural analgesia was not routinely administered, whereas opioid patient-controlled analgesia (PCA) was used systematically in both hospitals. There was no strategy to avoid opioids in the postoperative period. In the ERAS group, postoperative analgesia was systematically ensured using a mid-thoracic epidural or opioid PCA in cases of contraindication. Besides, the major difference was the systematic use of lidocaine infusions intra-operatively to

karger@karger.com

(c) 2020 S. Karger AG, Basel

www.karger.com/dsu

Karger! reduce opioid use. Finally, in the non-ERAS cohort including the control hospital, $60 \%$ of the patients underwent thoracic epidural, while almost $40 \%$ received PCA. Results were comparable between the two hospitals. In the ERAS group, $80 \%$ of the patients underwent thoracic epidural, while $25 \%$ received opioid PCA. In both hospitals, NSAIDs were not used. Finally, we can conclude that management of perioperative pain differed only in the ERAS group with an opioid-sparing strategy as recommended in the recent guidelines [3]. There was no impact on the final results.

In the second comment, Dr. Malgieri reported that we did not provide evidence that the time-to-event data are proportional and this may alter the interpretation of the results. However, the main outcome of the study was postoperative length of stay defined from the date of surgery to the date of discharge from the surgical department. Besides, secondary outcomes were postoperative morbidity, re-intervention rate, and postoperative mortality; data on these were all collected within 30 days after the surgery. Time-to-event data were comparable between the patients. Finally, we used a difference-in-differences Cox regression to evaluate the impact of the ERAS program considering the different groups: ERAS/nonERAS and before/after. It is different from the Cox regression analysis used for survival studies. 


\section{Disclosure Statement}

The authors have no conflicts of interest to declare.

\section{Funding Sources}

The authors did not receive any funding.

\section{References}

1 Perinel J, Duclos A, Payet C, Bouffard Y, Lifante JC, Adham M. Impact of enhanced recovery program after surgery in patients undergoing pancreatectomy on postoperative outcomes: a controlled before and after study. Dig Surg. 2019 Feb;37(1):47-55.

2 Lassen K, Coolsen MM, Slim K, Carli F, de Aguilar-Nascimento JE, Schäfer M, et al. Guidelines for perioperative care for pancreaticoduodenectomy: Enhanced Recovery After Surgery $\left(\right.$ ERAS $\left.^{\circledR}\right)$ Society recommendations. Clin Nutr. 2012 Dec;31(6):817-30.

3 Melloul E, Lassen K, Roulin D, Grass F, Perinel J, Adham M, et al. Guidelines for perioperative care for pancreatoduodenectomy: enhanced recovery after surgery (ERAS) recommendations 2019. World J Surg. 2020. 